\title{
Mycological Profile of Chronic Rhinosinusitis
}

\author{
V.A. Vipula ${ }^{1}$, G. Shobha Latha $^{1 *}$, S. Radha $^{1}$ and R.S. Pushpa Kumari ${ }^{2}$ \\ ${ }^{1}$ Department of Microbiology, ${ }^{2}$ Department of General Medicine, MNR Medical College and \\ Hospital, Sangareddy, Telangana, India \\ *Corresponding author
}

\section{Keywords}

Aspergillus flavus, Chronic rhinosinusitis, Fungal rhinosinusitis, $\mathrm{H}$ and $\mathrm{E}$ stain

Article Info

Accepted:

20 January 2018

Available Online:

10 February 2018

\section{A B S T R A C T}

Aim of the to assess the purpose of fungal rhinosinusitis at MNR Medical College \& Hospital, Sangareddy, and to correlate histopathological findings with culture results for accurate clinical classification of the disease. 217 suspected patients were included in the study. The relevant clinical details of the patients including the co-morbidities in the medical records were examined. Samples like nasal swabs collected during nasal endoscopy under sterile conditions, Sinus washings, Allergic mucin, tissue biopsy from polyps \& tissue biopsy taken from sinus mucosa during nasal surgery, were processed and examined by microbiology culture using recommended techniques. Slide culture was done to observe the microscopic morphology. Histopathological examination was done by $\mathrm{H}$ and E stain and PAS stain for classification. Out of 217 cases of rhinosinusitis, 37 samples showed fungal isolates in culture. Aspergillus flavus was the most common isolate (8.29\%). Aspergillus fumigatus was the second most common isolate (5.99\%). Candida albicans was isolated in one sample and candida species, other than albicans was isolated in one more sample. On the basis of the histopathological findings, the fungal isolates were grouped. Among the 37 patients with fungal rhinosinusitis 26 patients belonged to noninvasive type. They were mostly of allergic etiology. The organisms were mostly Aspergillus fumigatus and Aspergillus flavus. Isolates that cause fungal ball were not isolated. 11 isolates belonged to invasive group. Most of them were Aspergillus flavus causing chronic granulomatous fungal sinusitis Mycological profile of rhinosinusitis at MNR Medical College, Sangareddy was thus evaluated. Histopathological and microbiological findings reported 37cases of fungal rhinosinusitis among 217 suspected

\section{Introduction}

Chronic rhinosinusitis (CRS), defined as infection of the sinuses lasting for more than 3 months, is one of the most prevalent chronic illnesses in India affecting persons of all age groups. It is generally a mild disease. However, it is important to realize that it afflicts a significant percentage of the population, and causes considerable long term morbidity. Many patients with chronic rhinosinus disease are subjected to multiple courses of antibiotics and surgeries, with little or no improvement in their condition. Despite the tremendous advances in medicine over the last few decades, there have been relatively few advances in the diagnosis and treatment of chronic sinus disease. Long-term results of 
medical and surgical therapies have resulted in cure rates that vary between 29 and $80 \%$ (Hamaguchi et al., 1986; Melen et al., 1986; Murray and Jackson, 1983). We feel that this lack of progress is largely due to the paucity of knowledge on the microbiology and histopathology of chronic sinus disease available to us, and this was the impetus for our study.

Rhinosinusitis occurs in both acute and chronic forms, and represents a potential heterogeneity of pathophysiologies and prognosis. Chronic Rhinosinusitis accounts for more than $90 \%$ of all cases of Rhinosinusitis, has a slow protracted course, and has different etiologies, bacterial and fungal infections being a major cause.

A deeper understanding is thus critical, for Otolaryngorhinologists to move from an empiric decision making process, to a more evidence-based or culture-directed therapy paradigm. We undertook this study to prospectively examine the fungal etiology of chronic rhinosinusitis and for accurate clinical classification of the disease.

\section{Aims and Objectives}

The aim of the study was:

To study the spectrum of Fungal etiological agents among patients clinically diagnosed as Chronic rhinosinusitis and attending the ENT Outpatient Department MNR Medical College and Hospital Sangareddy, by:

Subjecting specimens collected from these patients to microscopy and to fungal culture.

Identifying the organism isolated.

To study the predisposing factors associated with the range of etiological agents isolated in culture.

\section{Inclusion criteria}

All patients with chronic rhinosinusitis diagnosed on the basis of clinical and radiological findings.

Allergic rhinitis patients with chronic sinusitis.

Patients above 10 years of age, both males and females were included.

\section{Exclusion criteria}

The patients with acute sinusitis, malignancy of paranasal sinuses were excluded from study.

\section{Materials and Methods}

This study was conducted at MNR Medical College and Hospital, Sangareddy, Medak Dist., for a period of one year from March 2012 to June 2013. Specimen processing was done in the Department of Microbiology, MNR Medical College and Hospital, Sangareddy. A total of 217 specimens were collected from patients suffering from Chronic rhinosinusitis attending the ENT Outpatient department of MNR Hospital Sangareddy from 1-11-2011 to 1-8-2013. The relevant clinical details of the patients including the comorbidities in the medical records were examined. These patients underwent a rigid nasal endoscopy with swabs and biopsies from the middle meatus to assess fungal etiology. CT scans of the paranasal sinus were performed to look for bone erosion and heterogeneous soft tissue opacity if any. Majority of these patients were not responding to conservative line of management with antibiotics.

\section{Specimens included}

Nasal swabs collected during nasal endoscopy under sterile conditions, Sinus washings, 
Allergic mucin, collected during nasal resection, Tissue biopsy from polyps and Tissue biopsy taken from sinus mucosa during nasal surgery.

\section{Lab methods for isolation and identifiction of fungi}

\section{Macroscopic examination}

Samples were inspected for colour, odour and whether they were purulent, blood stained (during surgery), muco purulent, mucoid or clear.

\section{Microscopic examination}

A $\mathrm{KOH}$ preparation of the test material was made on a slide using $10 \% \mathrm{KOH}$. The slide was examined for the presence or absence of hyphae \& budding yeast cells. A second smear was made on a slide, stained with Gram's stain and examined for Gram positive budding yeast cells if any.

\section{Culture media used}

The fungal samples were inoculated with a loop onto the following media:

2 tubes of Sabouraud's Dextrose with chloramphenicol.

Corn meal agar for Candida species.

Preparation of Sabouraud's dextrose agar: The Himedia Sabouraud's dextrose agar was dissolved in distilled water by gentle heating. $\mathrm{pH}$ was adjusted to 5.4. It was sterilized in the autoclave at $121^{\circ} \mathrm{C}$ for $15 \mathrm{~min}$ at $15 \mathrm{lbs}$ pressure and $20 \mathrm{ml}$ was poured into sterile petridishes.

Preparation of corn meal agar: The Himedia corn meal agar was dissolved in distilled water by gentle heating. It was sterilized in the autoclave at $121^{\circ} \mathrm{C}$ for $15 \mathrm{~min}$ at $15 \mathrm{lbs}$ pressure and $20 \mathrm{ml}$ was poured into sterile petridishes.

1 tube of SDA with chloramphenicol was incubated at $37^{\circ} \mathrm{C}$ and the other tube was incubated at room temperature. Both tubes were examined on alternate days for four weeks and colony characters were noted. A portion of the colony was picked up with a straight wire, mounted on a slide with Lactophenol cotton blue solution. It was teased with a pair of teasing needles and the fungus was identified by its morphology. A slide culture was also done for confirmation.

\section{Slide culture preparation}

A sterile microscopic slide was placed on bent glass rod at the bottom of petri dish. A piece of one square centimeter block of Cornmeal agar was put on the slide. Fungal strain was inoculated at four sides of agar block. The inoculated block was covered with sterile coverslip and incubated at $25^{\circ} \mathrm{C}$ in $\mathrm{BOD}$ incubator. A little sterile distilled water was added on filter paper to avoid drying of agar. When growth appeared, a drop of LCB was placed on slide and coverslip from block and examined microscopically to identify the fungus (Sanam Jindal, 2013).

A Gram's stain was also done where indicated, and examined for Gram positive budding yeast cells, indicative of Candida species.

Specimens that were positive for Candida were further subjected to Germ tube test. 0.5 $\mathrm{ml}$ of serum was taken in a test tube and was inoculated with small portion of colony from Sabouraud's dextrose agar growth and incubated at $37^{\circ} \mathrm{C}$ in a water bath for 2 hours. One loopful of serum yeast culture was taken on to a microscopic slide and examined for the formation of pseudohyphae. 
The colonies were also inoculated on cornmeal agar and incubated at $25^{\circ} \mathrm{C}$ for 72 hours. Abundant branched pseudohyphae and true hyphae with blastoconidia were noted. Terminal chlamydoconidia were formed with extended incubation.

Fungal isolates were diagnosed and grouped depending on the histopathological reports.

\section{Statistical analysis}

Statistical analysis of data was recorded and analysed using SPSS version 11.5 and the study was evaluated using $\mathrm{CHI}$ square test.

\section{Results and Discussion}

Among the 217 pts, who presented with CRS, there were 0 pts., in the age group 0-10yrs., 27 $(12.44 \%)$ between 11-20yrs., 56(25.80\%) between 21-30 yrs., 54(18.43\%) between 3140yrs, 37 (17.05\%) between 41- 50 years, 20 $(9.25 \%)$ between $51-60$ years and 23 (10.59\%) pts, above 60 years. The maximum number of pts., included in our study was in the 21- 30 years age group followed by 3140yrs age group. The age incidence in the present study is shown in Table.1.

Table. 2 shows age incidence and culture positivity. Maximum fungal culture positivity (20\%) was seen in the age group 51-60 years.

Among 46 pts., who had nasal allergy, fungal growth was seen in 6 pts., and Among 18 pts., with Diabetes mellitus fungal growth was seen in 5 pts. Among 41 pts with dental caries, 6 had fungal growth 37 smokers 8 positive for fungus and among 6 swimmers were -ve for fungal growth as shown in table 3 .

The most common symptom was nasal discharge in $76.03 \%$ with $20 \%$ fungal culture positivity. In pts with nasal block 21.77 were positive for fungus. Among pts with head ache
$18.42 \%$ had fungal growth. In patients with foul smelling breath $20.45 \%$ had fungal growth as shown in Table 4.

Among 116 pts., with deviated nasal septum, out of 217, positive fungal cultures in 29 cases. Among 5 pts., with polyp, 2 had fungal growth. And among 138 pts with hypertrophied turbinate, 25 were positive for fungus as shown in Table 5.

Out of 37 samples that showed fungal isolates in culture, Aspergillus flavus was the most common isolate, seen in $18(8.29 \%)$ out of 217 specimens. Aspergillus fumigatus was seen in 13 (5.99\%) out of 217 specimens, Candida albicans in $1(0.46 \%)$, Non Candida albicans in $1(0.46 \%)$ specimens, Mucor species in $2(0.92 \%)$, and Penicillium species was seen in $2(0.92 \%)$ out of 217 specimens as shown in Table.6.

Among the 37 patients with fungal rhinosinusitis 26 patients belonged to noninvasive type. The organisms were Aspergillus fumigatus 12, Aspergillus flavus 10, Candida albicans 1 and Non Candida albicans 1. Isolates that cause fungal ball were nil.11 isolates belonged to invasive group. They were Aspergillus flavus 8 isolates, Aspergillus fumigatus 1 and Mucor species 2 shown in table 7.

Among 217 specimens, nasal swabs collected during endoscopy were 102 with 9 showing fungal growths. Among 87 sinus washings samples, 5 were positive for fungus. Among the 14 tissue biopsies collected from sinus mucosa, 13 had fungal growth. Among the 5 polyps 5 had fungal growth. This is shown in Table.8. Number of fungal positive cultures in, Type II Diabetes mellitus cases is shown in table 9. Aspergillus fumigatus was isolated in 1 (5.55\%), Mucor spp. In 2 (11.11\%), Candida albicans in 1 case $(5.55 \%)$ and noncandida in $1(5.55 \%)$ case. 
Table.1 Age Incidence

\begin{tabular}{|l|}
\hline Age in years \\
\hline $0-10$ \\
\hline $11-20$ \\
\hline $21-30$ \\
\hline $31-40$ \\
\hline $41-50$ \\
\hline $51-60$ \\
\hline Above 60 (61-83) \\
\hline
\end{tabular}

\begin{tabular}{|l|l|}
\hline No. of pts. tested & \% of pts. tested \\
\hline- & - \\
\hline 27 & 12.44 \\
\hline 56 & 25.80 \\
\hline 54 & 18.43 \\
\hline 37 & 17.05 \\
\hline 20 & 9.25 \\
\hline 23 & 10.59 \\
\hline
\end{tabular}

Mean age: $36.5 \mathrm{yrs}$.

Table.2 Age incidence and culture positivity

\begin{tabular}{|l|l|}
\hline $0-10$ \\
\hline $11-20$ \\
\hline $21-30$ \\
\hline $31-40$ \\
\hline $41-50$ \\
\hline $51-60$ \\
\hline Above 60 \\
\hline
\end{tabular}

\begin{tabular}{|l|l|l|l|}
\hline No. of cases tested & $\%$ & Fungal culture +ve & $\%$ \\
\hline- & - & - & - \\
\hline 27 & 12.44 & 5 & 18.51 \\
\hline 56 & 25.80 & 11 & 19.64 \\
\hline 54 & 18.43 & 8 & 14.81 \\
\hline 37 & 17.05 & 5 & 13.51 \\
\hline 20 & 9.25 & 4 & 20 \\
\hline 23 & 10.59 & 4 & 17.39 \\
\hline
\end{tabular}

Chi-Square Value $=16.681 \mathrm{DF}=5 \mathrm{P}-$ Value $=0.005$

There is Significant association among the age group.

Table.3 Predisposing factors- culture positivity

\begin{tabular}{|l|r|l|r|r|}
\hline PREDISPOSING FACTOR & TOTAL & $\begin{array}{l}\text { FUNGAL } \\
\text { ISOLATE }\end{array}$ & \multicolumn{1}{l|}{$\%$} \\
\hline Nasal allergy & 46 & 6 & $13.04 \%$ \\
\hline Dental caries & 41 & 6 & 14.63 \\
\hline Smoking & 37 & 8 & $20 \%$ \\
\hline DM & 18 & 5 & $27.77 \%$ \\
\hline Swimming & 6 & 0 & $0 \%$ \\
\hline
\end{tabular}

Table.4 Symptoms-culture positivity

\begin{tabular}{|l|l|l|l|}
\hline $\begin{array}{l}\text { Clinical presentation } \\
\text { (symptom) }\end{array}$ & Total No. & $\begin{array}{l}\text { Fungal culture } \\
\text { +ve }\end{array}$ & $\%$ \\
\hline Nasal discharge & 165 & 33 & 20 \\
\hline Nasal block & 124 & 27 & 21.77 \\
\hline Head ache & 114 & 21 & 18.42 \\
\hline Foul smell & 44 & 9 & 20.45 \\
\hline
\end{tabular}


Table.5 Clinical finding - culture positivity

\begin{tabular}{|l|c|c|c|}
\hline NASAL OBSTRUCTION & TOTAL & FUNGAL CULTURE + VE & $\%$ \\
\hline POLYP & 5 & 2 & $40 \%$ \\
\hline DNS & 116 & 29 & $25 \%$ \\
\hline TURBINATE HYPERTROPHY & 138 & 25 & $18.11 \%$ \\
\hline
\end{tabular}

Chi-Square Value $=88.62$ DF=2 P-Value $=0.000$

There is Significant Accosiation of Clinical finding

Table.6 Fungal isolates from 217 cases of CRS

\begin{tabular}{l|}
\hline FUNGAL ISOLATE \\
\hline Aspergillus flavus \\
\hline Aspergillus fumigatus \\
\hline Mucor spp \\
\hline Penicillium spp \\
\hline Candida albicans \\
\hline Non Candida albicans \\
\hline
\end{tabular}

\begin{tabular}{|c|c|}
\hline NUMBER & $\%$ \\
\hline 18 & $8.29 \%$ \\
\hline 13 & $5.99 \%$ \\
\hline 2 & $0.92 \%$ \\
\hline 2 & $0.92 \%$ \\
\hline 1 & $0.46 \%$ \\
\hline 1 & $0.46 \%$ \\
\hline
\end{tabular}

Table.7 Types of fungal isolates depending on histopathology

\begin{tabular}{|c|c|c|c|}
\hline HISTOPATHOLOGY & $\begin{array}{l}\text { FUNGAL } \\
\text { GROWTH }\end{array}$ & $\begin{array}{l}\text { TYPE OF FUNGAL } \\
\text { SINUSITIS }\end{array}$ & $\begin{array}{l}\text { NO. OFCULTURE } \\
+ \text { VE PATIENTS }\end{array}$ \\
\hline \multicolumn{4}{|l|}{ NON INVASIVE } \\
\hline \multirow[t]{2}{*}{ 1)allergic mucin +ve } & \multirow{6}{*}{$\begin{array}{l}\text { Aspergillus } \\
\text { fumigatus } \\
\text { Aspergillus flavus } \\
\text { Candida albicans } \\
\text { Non Candida } \\
\text { albicans } \\
\text { Penicillium spp }\end{array}$} & \multirow{6}{*}{$\begin{array}{l}\text { ALLERGIC } \\
\text { FUNGAL SINUSITIS }\end{array}$} & 12 \\
\hline & & & 10 \\
\hline \multirow{2}{*}{$\begin{array}{l}\text { 2)degenerated } \\
\text { eosinophils +ve } \\
\end{array}$} & & & 1 \\
\hline & & & 1 \\
\hline $\begin{array}{l}\text { 3)segmental } \\
\text { branching + ve }\end{array}$ & & & \multirow[t]{2}{*}{2} \\
\hline 4)hyphae +ve & & & \\
\hline 5)fungal balls & Nil & FUNGAL BALL & ---------------- \\
\hline \multicolumn{4}{|l|}{ INVASIVE } \\
\hline Acute fulminant & Mucor species & $\begin{array}{l}\text { ACUTE } \\
\text { FULMINANT } \\
\text { FUNGAL SINUSITIS }\end{array}$ & 2 \\
\hline Granuloma + & A. flavus & $\begin{array}{l}\text { CHRONIC } \\
\text { INVASIVE } \\
\text { GRANULOMATOUS } \\
\text { SINUSITIS }\end{array}$ & 8 \\
\hline No granuloma & A. fumigatus & $\begin{array}{l}\text { CHRONIC } \\
\text { INVASIVE NON } \\
\text { GRANULOMATOUS } \\
\text { FUNGAL SINUSITIS }\end{array}$ & 1 \\
\hline
\end{tabular}


Table.8 Specimens with culture positivity

\begin{tabular}{|l|r|r|r|}
\hline SPECIMEN & TOTAL & FUNGAL ISOLATES \\
\hline Nasal swab & 102 & 9 & $8.82 \%$ \\
\hline Sinus washings & 87 & 5 & $5.74 \%$ \\
\hline Tissue biopsy from sinus mucosa & 14 & 13 & $92.85 \%$ \\
\hline Allergic mucin & 6 & 5 & $83.33 \%$ \\
\hline Polyp & 5 & 5 & $100 \%$ \\
\hline Throat swab & 3 & 0 & $0.00 \%$ \\
\hline
\end{tabular}

Chi-Square Value $=168.02$ DF=5 P-Value $=0.000$

There is significance between Specimens and culturePositivity

Table.9 Type II DM with Fungal Culture Positivity

\begin{tabular}{|l|l|l|}
\hline Aspergillus fumigatus & 1 & $5.55 \%$ \\
\hline Mucor spp & 2 & $11.11 \%$ \\
\hline Candida albicans & 1 & $5.55 \%$ \\
\hline Non Candida albicans & 1 & $5.55 \%$ \\
\hline
\end{tabular}

Rhinosinusitis is a common medical problem encountered in patients attending the Department of Otorhinolaryngology. Rhinosinusitis can be acute or chronic, classified according to the duration of symptoms. Acute rhinosinusitis lasts upto 12 weeks with complete resolution of symptoms, whereas the chronic form persists beyond 12 weeks. Patients with acute sinusitis present with fever, head ache, common cold and other complaints relating to oral or nasal infections. In the chronic form, fever may be low grade. Patients may present with nasal stuffiness, nasal discharge (of any character from thin to thick and from clear to purulent), postnasal drip, facial fullness, discomfort, headache, chronic unproductive cough, hyposmia, sore throat, fetid breath, malaise and easy fatigability.

The etiology, pathogenesis and management of CRS have been one of the most controversial topics in Otolaryngology. The literature available regarding this is sparse and difficult to interpret. In the present study an attempt was made to study the predisposing factors, to examine the fungal etiology of CRS. 217 patients who were clinically diagnosed as Chronic rhinosinusitis, that attended the Department of ENT, MNR Medical college and Hospital Sangareddy, were studied over a period of 1 year and eight months from 1-11-2011 to $1-8-2013$.

Endoscopic specimens from these patients were subjected to microscopy, fungal culture. Those that showed fungal elements on microscopy were also subjected to histopathological examination. The specimens examined were nasal swabs collected during nasal endoscopy, sinus washings, allergic mucin collected during nasal resection, tissue biopsy from polyps and tissue biopsy taken from sinus mucosa during nasal surgery.

In our study the common age group of CRS was from 20-70 years. High fungal culture positivity was seen in the age group 51-60 years (20\%) followed by 21-30years age group (19.64\%). It is similar to the study done by (Prateek et al., 2013).

In the present study male population affected were $55.76 \%$ among the 217 patients studied and their culture positivity was $19 \%$ for fungal infections. The female populations 
affected were $44.23 \%$ with fungal culture positivity of $14.58 \%$. There is slight male dominance in the incidence of CRS or in the positivity of the cultures in our study, although less significant than the male dominance reported in studies by (Prateek et al., 2013) and (Shilpa K. Gokale et al., 2010).

In the present study the rural population is high i.e. 158 out of 217 with $18.35 \%$ of them being fungal culture positive. The urban population is 59 with $13.55 \%$ fungal positivity showing predominance of fungal infections in the rural population which is similar to the other studies from Sudan and North India (Chakrabarti et al., 1992).

Among all the predisposing factors studied, like nasal allergy, dental caries, diabetes mellitus, smoking and swimming, the most common predisposing factor was found to be nasal allergy (21.19\%) followed by dental caries $(18.89 \%)$ in our study. The values are similar when compared with the studies done by Shapira (1985), Turner et al., for nasal allergy and studies of Melen and colleagues for dental caries.

The most common pathological finding in CRS patients is nasal obstruction in our study. Among 217 patients studied, 138 patients had turbinate hypertrophy, 116 patients had deviated nasal septum and 5 patients had nasal polyps. In all three conditions fungal culture positivity was 18-25\%. Itzahk Brook et al., (1982), Berry 1930 reported nasal obstruction as a cause of CRS in $54.54 \%$ of cases. It correlates with our study where nasal obstruction is present in more than $50 \%$ of cases. This is the commonest pathological predisposing factor described for CRS.

The common presenting symptom in our study group was nasal discharge with bacterial culture positivity of $73.33 \%$ followed by foul smelling breath with bacterial culture positivity (72.72\%). This is comparable with most of the other studies. Nasal block, on the other hand, was the commonest symptom in fungal sinusitis.

Among all the 217 patients in our study, aerobic fungal growth was positive in $17.05 \%$. In a study by (Panduranga Kamat et $a l ., 2013$ ) the fungal growth was $7.4 \%$. When compared with these studies, our study showed higher prevalence for fungal growths. This shows the high prevalence of fungal infections in our study area.

Out of 37 samples that showed fungal isolates in culture, in our study, Aspergillus flavus was the most common isolate (8.29\%). Aspergillus fumigatus was the second most common isolate $(5.99 \%)$ similar to the studies of (Chakrabarti et al., 1992) and (Panda et al., 1998). Candida albicans was isolated in one sample and candida species, other than albicans was isolated in one more sample. Even though candidial infection is rare in CRS we presume the infection in our study may be due to the associated Diabetes mellitus in these patients. On the basis of the histopathological findings, the fungal isolates were grouped. Among the 37 patients with fungal rhinosinusitis 26 patients belonged to non-invasive type. They were mostly of allergic etiology. The organisms were mostly Aspergillus fumigatus and Aspergillus flavus. Isolates that cause fungal ball were not isolated.11 isolates belonged to invasive group. Most of them were Aspergillus flavus causing chronic granulomatous fungal sinusitis. Our results correlated well with the reports of Prateek et al., (2013).

Among the various specimens examined, nasal swabs taken during endoscopy were highest in number. Fungal isolates were more in tissue specimens like nasal polyps (100\%), followed by tissue biopsy from sinus mucosa $(92.85 \%)$ and allergic mucin $(83.33 \%)$. 
In our study among 217 patients with CRS 18 patients was Diabetics. Fungal isolates were $27.77 \%$ among them. When compared with other etiological factors, diabetes mellitus as a predisposing factor is less common. The infection rate is more in patients with diabetes mellitus.

From our study we conclude that the most common predisposing factors for CRS are nasal allergy, dental caries and nasal obstruction. 37 patients had fungal infections associated with CRS which is also a significant association. The common fungal infections are due to Aspergillus flavus and Aspergillus fumigatus. Hence, it is suggested that culture directed therapy is the gold standard for the management of CRS.

\section{References}

Chakrabarti A, Sharma SC, Chander J. Epidemiology and pathogenesis of paranasal sinus mycoses. Indian $\mathbf{J}$ Otorhinolaryngol Head Neck Surg 1992; 107:745- 50.

DeShazo RD, Chapin K, Swain RE. Fungal sinusitis. N Engl J Med1997; 337:254-9.

Ferguson BJ. Definitions of fungal rhinosinusitis. Otolaryngol Clin North Am., 2000; 33:227235.

Gwaltney JM Jr. Microbiology of sinusitis. In: Druce HM, editor. Sinusitis: pathophysiology and treatment. New York: Marcel Dekker; 1994. pp. 41-56.

Hamaguchi, Y., M. Ohi, Y. Sakakura, and Y. Miyoshi. 1986. Significance of lysosomal proteases; cathepsins $\mathrm{B}$ and $\mathrm{H}$ in maxillary mucosa and nasal polyp with non-atopic chronic inflammation. Rhinology, 24:187194
Melen, I., L. Lindahl, and L. Andreasson. 1986. Short and long-term treatment results in chronic maxillary sinusitis. Acta Otolaryngol. (Stockholm) 102:282-290.

Morgan MA, Wilson WR, Neil HB III, Roberts GD. Fungal sinusitis in healthy and immunocompromised individuals. Am J Clin Pathol1984; 82:597-601.

Murray, J. P., and M. S. Jackson. 1983. Complications after treatment of chronic maxillary sinus disease with Caldwell-Luc procedure. Laryngoscope 93:282-284.

Panda NK, Sharma SC, Chakrabarti A, Mann SB. Paranasal sinus mycosesin north India. Mycoses 1998; 41:281-6.

Panduranga Kamath, M., Vijendra Shenoy S, Nitin Mittal, nitish Sharma Microbiological analysis of paranasal sinuses in chronic sinusitis- A south Indian coastal study. Egyptian journal of Ear, Nose, Throat and Allied Sciences (2013)14.185, 189

Prateek S, Banerjee G, Gupta P, Singh M, Goel MM, Verma V. Fungal rhinosinusitis: A prospective study in a University hospital of Uttar Pradesh. Indian J Med Microbiol 2013; 31:266-9.

Sanam Jindal*, M. Panduranga Kamath Analysis Of Microbial Flora In Patients With Chronic Sinusitis Under Going FunctionalEndoscopic Sinus Surgery (FESS): A Cross-Sectional Study Int. J. Fundamental Applied Sci. Vol. 2, No. 1(2013) 2-4

Shilpa K Gokale, and Shashidhar S suligavi Bacteriological study of Chronic maxillary sinusitis with special reference to anaerobes. Clinical Rhinology: An international. Journal, September December 2010 3(3) 141- 144

Thrasher RD, Kingdom TT. Fungal infections of the head and neck: Anupdate. Otolaryngol Clin North Am 2003; 36:577-94.

\section{How to cite this article:}

Vipula, V.A., G. Shobha Latha, S. Radha and Pushpa Kumari, R.S. 2018. Mycological Profile of Chronic Rhinosinusitis. Int.J.Curr.Microbiol.App.Sci. 7(02): 2332-2340.

doi: https://doi.org/10.20546/ijcmas.2018.702.283 